INTERNATIONAL BULLETIN OF BACTERIOLOGICAL
NOMENCLATURE AND TAXONOMY
$\begin{array}{cccc}\text { Volume } 10 & \text { No. } 2 & \text { April 15, } 1960 & 71-74\end{array}$

\title{
A PROPOSED GENUS ENTEROBACTER
}

\author{
E. Hormaeche and P.R. Edwards \\ Instituto de Higiene, Montevideo, \\ Uruguay, and Communicable Disease \\ Center, Public Health Service, U.S. \\ Department of Health, Education and \\ Welfare, Atlanta, Georgia
}

It was demonstrated by Edwards (1) more than 30 years ago that cultures accepted as typical strains of Aerobacter aerogenes, and isolated under circumstances which did not indicate that they had any immediate connection with the intestinal tract of man or animals, were indistinguishable from Klebsiella cultures and, indeed, some of them were biochemically and serologically identical with classical Friedlander strains. In spite of this observation, and although the Judicial Commission has conserved the generic term Klebsiella Trevisan 1885 and the specific epithet Klebsiella pneumoniae (Schroeter) Trevisan 1885, such organisms when isolated from stools, urine, soil, and water, continue to be classified as $\mathrm{A}$. aerogenes. Most workers in systematic bacteriology would agree with the original description of Beijerinck (2) that the majority of strains of $\mathrm{A}$. aerogenes are nonmotile, yet no method has been devised to distinguish such strains from Klebsiella pneumoniae. Here we are faced with a paradoxical situation. The Judicial Commission has conserved Klebsiella pneumoniae but the majority of cultures which conform to the description of that species continue to be classified in the genus Aerobacter. In Bergey's Manual (3) both motile and nonmotile strains of A. aerogenes are recognized but no method is given whereby the nonmotile strains can be distinguished from strains of the genus Klebsiella. Capsules and slime formation are of no value in differentiation since both properties are found in both motile and nonmotile strains and may vary within a single culture. Although Breed, Murray and Smith (3) recognize this situation, they continue to place organisms indistinguishable from $K$. pneumoniae in the genus Aerobacter, giving as their reason the difficulties encountered by earlier workers in distinguishing between such organisms and motile forms classified as Aerobacter cloacae. Considering the 
present state of our knowledge of the biochemical properties of the two groups, such a standpoint hardly seems tenable.

It was the above-described situation which prompted Hormaeche and Edwards (4) to propose the genus Aerobacter be limited to forms which typically were motile and which could be distinguished from the genus Klebsiella by biochemical methods. Two species were described, A. aerogenes and $A$. cloacae. Inasmuch as the term Aerobacter has been so misused, and since this misuse probably will continue so long as the genus Aerobacter is recognized, it now seems best to withdraw this proposal and to seek another solution.

The generic name Cloacae Castellani and Chalmers1919, has not been conserved nor has it found wide use. Further, the name of the type species of Cloaca, C. cloacae, is almost a tautonym and should be rejected under Rule 25 of the Bacteriological Code. It would not be logical to conserve both Cloaca and Aerobacter, since the same organisms have been classified in both. We are faced either with continuing to recognize Aerobacter, the disadvantages of which have been enumerated, or of seeking a neutral designation.

The new generic name Enterobacter is proposed with type species Enterobacter cloacae (basionym Bacillus cloacae Jordon 1890). The name Enterobacter proposed by Rahn (1937) was not validly published and in consequence has no standing in nomenclature and cannot be cited as an earlier homonym of the newly proposed generic name Enterobacter. According to our present taxonomic knowledge, the nonmotile cultures hitherto designated as A. aerogenes, other than nonmotile variants of Enterobacter which can be distinguished by biochemical methods, must be classified in the genus Klebsiella, which has beenconserved. The motile forms previously designated as $A$. cloacae and $\underline{A}$. aerogenes would be included in the genus Enterobacter as E. cloacae or E. aerogenes according to their biochemical reactions which are defined below.

Enterobacter gen. nov.

Motile peritrichously flagellated rods conforming to the definition of the family Enterobacteriacae. Gram negative, grow readily on ordinary media. Ferment glucose and lac- 
B ACTERIOLOGICAL NOMENCLATURE

\section{AND TAXONOMY}

tose with the production of acid and gas. Produce two or more times as much carbon dioxide as hydrogen from glucose. Methyl red test negative; Voges-Proskauer test positive. Trimethyleneglycol not produced from glycerol by anaerobic fermentation. Citric acid and salts of citric acid are utilized as sole sources of carbon. Hydrogen sulfide is not produced. Aerobic, facultatively anaerobic. Widely distributed in nature. Nonmotile and anaerogenic variants occur which may be recognized by their otherwise typical biochemical behavior.

The type species is E. cloacae (Jordan 1890) Nov. Comb. The biochemical reactions of $\underline{E}$. cloacae and $\underline{E}$. aerogenes are given in Table 1 .

Typical neotype cultures have been deposited in the American Type Culture Collection and the National Collection of Type Cultures, London, under the following numbers: E. cloacae, 279-56 (ATCC 13047; NCTC 10005); E. aerogenes, 819-56 (ATCC 13048; NCTC 10006).

\section{REFERENCES}

1. Edwards, P.R. 1929. J. Bact. 17: 339 .

2. Beijerinck, M.W. 1900. Zbl. Bakt., II Abt. 6: 193.

3. Breed, R.S., E. G. D. Murray and N.R. Smith. 1957. Bergey's Manual of Determinative Bacteriology, Williams and Wilkins Co., Baltimore.

4. Hormaeche, E. and P.R. Edwards. 1958. Intérn.

Bull. Bact. Nomen. and Taxon. 8: 111 .

5. Rahn, O. 1937. Zbl. Bakt., I Abt. 96: 280.

6. Hormaeche, E. and M. Munilla. 1957. Intern. Bull. Bact. Nomen. and Taxon. 7: 1 .

\section{ACKNOW LEDGMENT}

The writers are indebted to Dr. F. Kauffmann for suggesting the term Enterobacter as a solution of the problem posed by continued application of the term Aerobacter to cultures which should be classified in the genus Klebsiella. 
Table 1. Biochemical Characteristics

Enterobacter Enterobacter aerogenes cloacae

Gas from glucose

Lactose (acid)

Sucrose

Mannitol

Dulcitol

$\begin{array}{ll}+ & + \\ + & + \\ + & + \\ + & + \\ \mathrm{d} & \mathrm{d}\end{array}$

Salicin

Adonitol

Inositol (gas)*

Glycerol (gas)*

Rhamnose

$+$

$+$

d

Raffinose

Arabinose

Xylose

Sorbitol

Cellobiose (gas)*

Insoluble starch (gas)**

Indol

Methyl red

Voges-Proskauer

Ammonium citrate

Hydrogen sulfide

Urease***

Gelatin liquefaction

Growth in KCN medium

Lysine decarboxylase

Arginine dihydrolase

Ornithine decarboxylase

Glutamic acid decarboxylase Motility

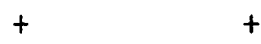

d d

+ -

$+\quad-$

$+\quad+$

$+\quad+$

$+\quad+$

$+\quad t$

$+\quad+$

$+\quad+$
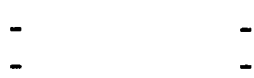

$-$

$+\quad+$

$+\quad+$
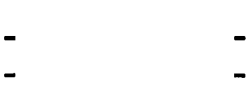

d $\quad+$

$+\quad+$

$+\quad-$

$-\quad+$

$+\quad+$

$-$

$+\quad+$

* Visible gas production within 48 hours.

* Visible gas production within 4 days.

*** Tested by method of Hormaeche and Munilla (6).

+ Promptly positive.

- Negative.

d Different biochemical types. 